\title{
A NEW TRELLIS REPRESENTATION FOR SOURCE-CHANNEL RATE ALLOCATION
}

\author{
Romain Tajan, Charly Poulliat, Rodrigue Imad and Inbar Fijalkow \\ ETIS - CNRS / ENSEA / UCP, 6 Avenue du Ponceau \\ 95000 Cergy-Pontoise, France
}

\begin{abstract}
In this paper, we consider the source-channel rate allocation for different transmission schemes. We propose a new trellis structure and a new algorithm that are able to deal with both variable length packet and fixed length packet problems. The trellis description allows to handle any kind of transmission schemes and can therefore be applied to Bit Interleaved Coded Modulations (BICM), parallel channels or Hybrid-Automatic Repeat reQuest (HARQ).
\end{abstract}

Index Terms - source-channel coding, rate allocation algorithm, trellis, Unequal Error Protection (UEP), Bit Interleaved Coded-Modulation (BICM)

\section{INTRODUCTION}

$S^{\prime}$ NCE the pioneer work by Sherwood and Zeger [1], progressive image transmission over error-prone channels has been widely investigated and studied for different transmission schemes and under different system assumptions. In this context, wavelet-based source-coders are generally considered, such as the Set Partitioning In Hierarchical Tree (SPIHT) [2] or JPEG-2000 [3]. These sourcecoders can generate embedded bit-streams that allow the progressive reconstruction of the source at different bit rates from the prefix of a single bit-stream. However, the generated bit-streams are usually very sensitive to errors. Therefore, many works have proposed combined source-channel allocation strategies to fully exploit the progressive nature of the embedded bit-stream. Considering packetbased coded transmissions using error detection based on Cyclic Redundancy Check (CRC) codes, the proposed schemes finally aim at providing Unequal Error Protection (UEP) for source packets to ensure proper reconstruction of the encoded bit-stream at the receiver. An UEP allocation strategy consists in choosing different code-rates for the source-packets according to some performance criterion under a total rate or bandwidth constraint for example. Thus, independently of system assumptions, there are mainly two types of combined source-channel allocation strategies considered in the literature, namely distortion-based and rate-based allocation strategies. In the first case, the allocation strategy is based on the minimization of a cost function based on the average distortion (ie. average Mean Square Error or average Peak Signal-to-Noise Ratio), assuming the knowledge or an estimation of the Rate-Distortion (R-D) curve. In the second case, a sub-optimal approach is considered using the maximization of the useful source coding rate, ie. the average number of correctly received source bits. For both strategies, two different system approaches can be considered for packetizing the source output. In the first approach, as originally introduced by [4], the length of the channel coded packets is variable while the length of the source packets remains constant. We refer to this allocation problem as the Variable-length Packet Problem (VPP). In the second approach, the length of the channel coded packets is kept constant.
We refer to this allocation problem as the Fixed-length Packet Problem (FPP).

In the case of binary inputs memoryless channels, these allocation problems were originally solved by [4] for the VPP case and by $[5,6]$ for the FPP case. Since then, numerous contributions considered possible extensions to other types of channels and coding schemes (either for the VPP or for the FPP case), or addressed complexity issues with regards to the distortion-based strategy in both VPP and FPP cases. In the VPP context, Chande and Farvardin proposed a dynamic programming based method to find an optimal UEP allocation strategy (also called policy) for the distortion-based and rate-based problems [4]. The method is based on a recursive equation that builds the optimal policy from the last packet to the first. In [5], Hamzaoui et al. developed an optimal rate-based allocation based on the recursion proposed in [4] and adapted to the FPP context. In [6], they proposed a sub-optimal distortion-based algorithm called the local search algorithm. Other sub-optimal approaches that were developed in $[7,8]$ use Viterbi-like algorithm on tree-like description structures. In addition, other extensions were considered to parallel channels in [9], space-time coded OFDM based MIMO in [10] and Hybrid Automatic Repeat reQuest (HARQ) in [11].

In this work, the rate-based allocation problem is considered. We propose a new regular trellis structure on which efficient computation of the solution of the rate-based allocation problem is done for both VPP and FPP problems. The trellis structure allows to switch easily between the VPP and the FPP and to easily compute the complexity of the algorithm. Moreover, due to the definition of the states involved in the trellis, extensions to spectrally efficient transmission schemes such as the adaptive Bit-Interleaved CodedModulation (BICM) or to transmission schemes considering HARQ are easily handled. This allows to consider a general framework for the rate-based allocation problem that can be considered for any kind of transmission scheme and any packetization strategy.

The paper is organized as follows. In Section 2, we present the general context of the study and give the main assumptions and notations used throughout the paper. In Section 3, we first describe the new trellis structure used and then give the description of our algorithm based on this trellis. Some optimization and simulation results are presented in Section 4. A conclusion and some perspectives are drawn in Section 5.

\section{PROBLEM STATEMENT}

In this paper, we consider the transmission of the packetized binary output of an embedded source coder over a memoryless channel, such as the Additive White Gaussian Noise (AWGN) channel. The protection of the source is based on the concatenation of an inner binary error-correcting code for error correction and an outer Cyclic Redundancy Check (CRC) for error detection. The CRC is considered to be perfect and detects errors with a probability of 
one. For practical reasons, we also consider that channel codes used for ensuring UEP are issued from a Rate Compatible (RC) family [12]. The output of the channel code is interleaved and then mapped to a $Q$-ary modulation with unit average energy per coded symbol $E_{s}$. Then, coded symbols are sent over the channel. Here, we consider the direct generalization of [4] and [5] for which the Binary Phase Shift Keing (BPSK) modulation was initially considered. The set of available rate-compatible channel codes is represented by $\mathcal{C}=\left\{c_{1}, \cdots, c_{N_{c}}\right\}$ where the $c_{i}$ are the channel codes indexed by increasing rate and $N_{c}$ is the number of available channel codes. The set of available $Q$-ary modulations is denoted by $\mathcal{M}=\left\{m_{1}, \cdots, m_{N_{m}}\right\}$ where the $m_{i}$ stands for the different modulations indexed by increasing spectral efficiency and $N_{m}$ is the number of possible modulations. Let the order of a modulation $m \in \mathcal{M}$ be denoted by $M(m)$. A BICM scheme is denoted by $c m_{i}=\left(c_{k_{i}}, m_{j_{i}}\right)$ where $c_{k_{i}} \in \mathcal{C}$ and $m_{j_{i}} \in \mathcal{M}$. The resulting set is noted $\mathcal{C M}$ and has a cardinality $N_{c m}=N_{c} N_{m}$. For a sourcepacket encoded by the BICM scheme $\mathrm{cm}_{i} \in \mathcal{C M}$, we denote by $k_{s}\left(\mathrm{~cm}_{i}\right)$ and $l\left(\mathrm{~cm}_{i}\right)$ the number of source bits in this source-packet and the number of bits at the output of the binary code, respectively. The resulting channel code rate is $r\left(c_{k_{i}}\right)=k_{s}\left(\mathrm{~cm}_{i}\right) / l\left(\mathrm{~cm}_{i}\right)$. Let $b\left(c m_{i}\right)=l\left(c m_{i}\right) / \log _{2}\left(M\left(m_{j_{i}}\right)\right)$ be the number of channel symbols at the output of the BICM scheme $\mathrm{cm}_{i}$, the rate in (source) bits per channel use of the BICM scheme $\mathrm{cm}_{i}$ is given by:

$$
R\left(c m_{i}\right)=r\left(c_{k_{i}}\right) \log _{2}\left(M\left(m_{j_{i}}\right)\right) .
$$

For the VPP case extended to BICM schemes, the number of information bits in a packet $k_{s}(\mathrm{~cm})$ is the same for all BICM schemes in the set $\mathcal{C} \mathcal{M}$. For the FPP case, the number of symbols in a channelpacket $b(\mathrm{~cm})$ is the same for all BICM schemes in $\mathcal{C} \mathcal{M}$. Then at a given signal-to-noise ratio $E_{s} / N_{0}$, we consider without loss of generality that $\mathcal{C M}$ verifies the following properties :

$$
\begin{aligned}
R\left(c m_{1}\right) & \leq R\left(c m_{2}\right) \leq \ldots \leq \quad R\left(c m_{N_{c m}}\right) \\
p_{e}\left(c m_{1}\right) & \leq p_{e}\left(c m_{2}\right) \leq \ldots \leq p_{e}\left(c m_{N_{c m}}\right) \\
k_{s}\left(c m_{1}\right) & \leq k_{s}\left(c m_{2}\right) \leq \ldots \leq \quad k_{s}\left(c m_{N_{c m}}\right) .
\end{aligned}
$$

The first assumption is related to the indexation of BICM schemes in $\mathcal{C} \mathcal{M}$ by increasing spectral efficiency. The second assumption is a natural assumption for a well-selected set of BICM schemes. This is related to the relative performance of BICM schemes. The third property can be considered as more restrictive but is verified in the VPP case extended to BICM schemes (for all BICM schemes, $k_{s}(\mathrm{~cm})$ is constant) and in the FPP case as well (for all coded-modulations, $b(\mathrm{~cm})$ is constant thus $k_{s}(\mathrm{~cm})=b R(\mathrm{~cm})$ so if $R\left(c m_{i}\right) \leq R\left(c m_{j}\right)$ we have $\left.k_{s}\left(c m_{i}\right) \leq k_{s}\left(c m_{j}\right)\right)$. Each source packet can be potentially encoded by a different BICM scheme. The allocation of a BICM scheme to a source-packet is done by a code allocation policy [4], which is denoted in this paper by $\Omega=\left(\mathrm{cm}_{\Omega}^{1}, \mathrm{~cm}_{\Omega}^{2}, \ldots c m_{\Omega}^{N_{\Omega}}\right) . N_{\Omega}$ represents the total number of source packets in the code allocation policy but can be also thought as the last index of the source-packets. The total symbol channel budget of a policy $\Omega$ is noted $B(\Omega)$ and is given by:

$$
B(\Omega)=\sum_{i=1}^{N_{\Omega}} b\left(c m_{\Omega}^{i}\right)
$$

The cost function evaluated in order to solve the rate-based sourcechannel allocation problem is the average number of source-bits cor- rectly received in a row using a policy $\Omega$. This quantity is given by:

$$
V(\Omega)=\sum_{i=1}^{N_{\Omega}}\left(\sum_{j=1}^{i} k_{s}\left(c m_{\Omega}^{i}\right)\right) P_{i}(\Omega),
$$

where $P_{i}(\Omega)$ represents the probability of receiving exactly $i$ packets in a row and is defined as:

$$
P_{i}(\Omega)=\prod_{l=1}^{i}\left(1-p_{e}\left(c m_{\Omega}^{l}\right)\right) p_{e}\left(c m_{\Omega}^{i+1}\right),
$$

with $p_{e}\left(c m_{\Omega}^{0}\right)=0$ and $p_{e}\left(c m_{\Omega}^{N_{\Omega}+1}\right)=1$.

The rate-based allocation problem is defined as follows:

$$
\max _{\Omega} V(\Omega) \text { subject to } B(\Omega) \leq B_{T}
$$

Given (4) and (5), the following recursion can be derived for all $i \in$ $\left[1, N_{\Omega}-1\right]$ as

$$
\begin{aligned}
& V\left(c m_{\Omega}^{i}, c m_{\Omega}^{i+1}, \ldots, c m_{\Omega}^{N_{\Omega}}\right)= \\
& \quad\left(1-p_{e}\left(c m_{\Omega}^{i}\right)\right)\left[k_{s}\left(c m_{\Omega}^{i}\right)+V\left(c m_{\Omega}^{i+1}, \ldots, c m_{\Omega}^{N_{\Omega}}\right)\right]
\end{aligned}
$$

Let $\Omega_{B_{T}}^{*}=\left(c m_{\Omega_{B_{T}}^{*}}^{1}, c m_{\Omega_{B_{T}}^{*}}^{2}, \ldots, c m_{\Omega_{B_{T}}^{*}}^{N_{B_{T}}^{*}}\right)$ be the optimal policy for the problem in (6), then $\Omega_{B_{T}}^{*}$ verifies the following proposition:

Proposition 1. Given the optimal policy $\Omega_{B_{T}}^{*}$ for the problem defined in (6) under the constraint $B_{T}$, then the policy $\Omega^{\prime}=$ $\left(\mathrm{cm}_{\Omega_{B_{T}}^{*}}^{2}, \ldots, \mathrm{cm}_{\Omega_{B_{T}}^{*}}^{N_{\Omega_{T}^{*}}^{*}}\right)$ is optimal for the maximization of $V\left(\Omega^{\prime}\right)$ (Eq. (6)) under the constraint $B\left(\Omega^{\prime}\right) \leq B_{T}-b\left(\mathrm{~cm}_{\Omega_{B_{T}}^{*}}^{1}\right)$.

Proof. The proof is a straightforward generalization of results in [4] for the VPP case and in [5] for the FPP case.

Let the solution of problem (6) be denoted by $V_{B_{T}}^{*}=V\left(\Omega_{B_{T}}^{*}\right)$. Considering proposition 1 and the recursion in (7), the following proposition can be stated:

Proposition 2. If $\Omega_{B_{T}}^{*}=\left(c m_{\Omega_{B_{T}}^{*}}^{1}, c m_{\Omega_{B_{T}}^{*}}^{2}, \ldots, c m_{\Omega_{B_{T}}^{*}}^{N_{B_{T}}^{*}}\right)$ is a rate-optimal policy under the constraint $B_{T}$ then $V_{B_{T}}^{*}$ verifies the recursion given in (8)

$$
V_{B_{T}}^{*}=\max _{c m \in \mathcal{C} \mathcal{M}}\left\{\left(1-p_{e}(c m)\right)\left[k_{s}(\mathrm{~cm})+V_{B_{T}-b(c m)}^{*}\right]\right\}
$$

\section{TRELLIS DESCRIPTION AND ASSOCIATED ALGORITHM}

\subsection{A new trellis description}

The trellis we introduce, is composed of sections that are indexed by the total symbol budget $B$ consumed to reach this section. For a section related to a budget $B_{0}$, each state is associated with a BICM scheme in the set $\mathcal{C M}$, ordered by increasing spectral efficiency. Branches start from a state $\mathrm{cm}_{i}$ of the section related to the budget $B_{0}$. They are linked with some other states of other sections. We denote by $\left(B, c m_{j}\right)$ the corresponding symbol budget and BICM scheme couples. The length of a branch going to a BICM scheme $\mathrm{cm}_{j}$ is equal to the symbol budget $b\left(\mathrm{~cm}_{j}\right)$ consumed when the coder $\mathrm{cm}_{j}$ is used. A path in the trellis represents a policy and the 


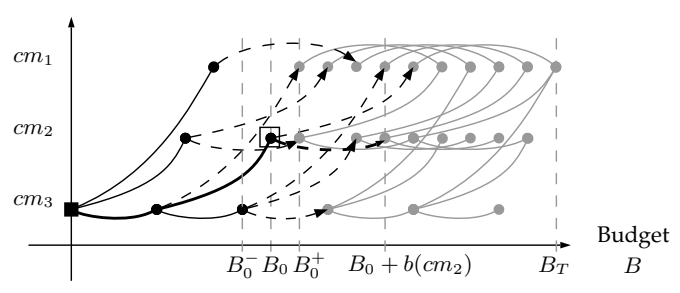

Fig. 1. VPP trellis structure for $\mathcal{C M}=\left\{c m_{1}, c m_{2}, c m_{3}\right\}$ with respective branches length $\{5,4,3\}$ being used by the presented algorithm in state $B_{0}$. Solid black branches and black states stand for kept policies. Dashed arrows are propagations done by the algorithm. Unvisited branches and states are represented by grey branches and grey states. The bold path represents the policy $\left(\mathrm{cm}_{3}, \mathrm{~cm}_{2}, \mathrm{~cm}_{2}\right)$.

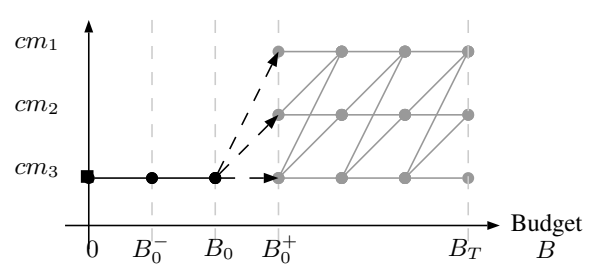

Fig. 2. FPP trellis structure (same legend as in Fig. 1).

length of this path is the budget consumed by this policy $B(\Omega)$. In order to reduce the complexity of the algorithm, the targeted policies are rate increasing. Considering the policy $\Omega=\left(\mathrm{cm}_{\Omega}^{1}, \ldots, \mathrm{cm}_{\Omega}^{N_{\Omega}}\right)$, it implies that if $i \leq j$ then $R\left(\mathrm{~cm}_{\Omega}^{i}\right) \leq R\left(\mathrm{~cm}_{\Omega}^{j}\right)$. In the trellis, this is shown by the fact that a state $\mathrm{cm}_{i}$ is only linked with states $\mathrm{cm}_{j}$ where $j \leq i$. We show in Section 3.2 that the complexity of the method is reduced but the optimality remains the same. As for [4] or [5], the policies are inherently built from the last packet to the first packet. As an illustration, a trellis is represented for a set of three different BICM schemes for both the VPP and FPP problems in Fig. 1 and Fig. 2, respectively.

\subsection{Algorithm description}

The trellis based allocation that we propose is given in Algorithm 1 . In this description, $B_{0}$ represents the current symbol budget, $B_{0}^{+}$the following symbol budget reached by the trellis and $B_{0}^{-}$the previous one. Note that those states are not necessarily linked. As we will see, for each section associated with a symbol budget $B$, the algorithm keeps only one state noted $\mathrm{cm}_{B}$. Thus it keeps only one policy starting from the root to $\mathrm{cm}_{B}$. This policy is noted $\Omega_{B}$.

In order to illustrate the behavior of Algorithm 1, let us describe what happens for a section of budget symbol $B_{0}$ that is known to be optimal. We suppose that all policies $\Omega_{B_{0}-b(\mathrm{~cm})}^{*}$ have already been computed, ie. the steps 7 to 10 have been done for all the sections $B_{0}-b(\mathrm{~cm})$ such as $R(\mathrm{~cm}) \leq R\left(\mathrm{~cm}_{\Omega_{B-b(\mathrm{~cm})}^{*}}^{1}\right)$. Then, Algorithm 1 computes the following recursion:

$$
\max _{\substack{c m \in \mathcal{M} \\ R(c m) \leq R\left(c m_{\Omega_{B-b(c m)}^{*}}^{1}\right)}}\left(1-p_{e}(\mathrm{~cm})\right)\left[k_{s}(\mathrm{~cm})+V\left(\Omega_{B-b(\mathrm{~cm})}^{*}\right)\right] .
$$

This recursion is equivalent to the recursion (8) to which we impose a rate increasing condition on the policies. This condition is a gener-

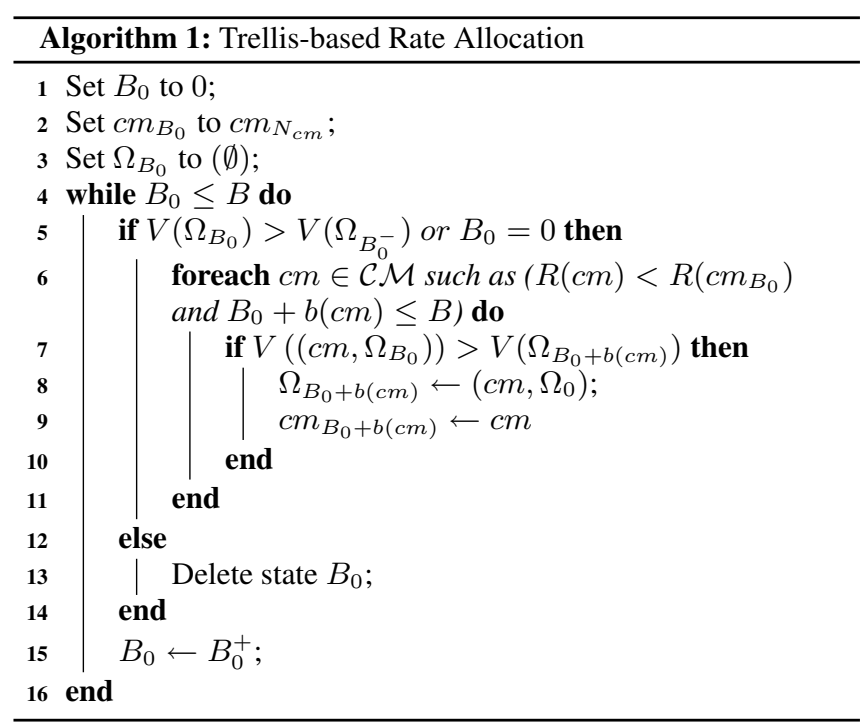

alization of the VPP case [4] for rate compatible policies and of the FPP case [5]. Although this condition seems restrictive, we give the following propositions (proofs are omitted due to lack of space):

Proposition 3. If a policy $\Omega$ is not rate increasing then it is possible to find a rate increasing policy which is at least as good as $\Omega$.

Proposition 4. Among all optimal policies of problem (6), at least one is rate increasing.

Propositions (3) and (4) imply that the rate increasing condition does not change the optimality of the method. Furthermore, the rate increasing condition restrains the enumeration in the computation of max, which improves the algorithm performance.

Let $G\left(B_{T}\right)=\left\{B(\Omega): B(\Omega)<B_{T}\right\}$ be the ensemble of reachable symbol budgets. The complexity of Algorithm 1 is at most $N_{c m}\left|G\left(B_{T}\right)\right|$ multiplications. Thus, the complexity of Algorithm 1 is similar to the ones in [4] for the VPP case and [5] for the FPP case. We can also show that the maximum number of sections that have to be stored to compute the rate-optimal policy is equal to $b\left(\mathrm{~cm}_{1}\right) / G C D(b(\mathrm{~cm}), \mathrm{cm} \in \mathcal{C} \mathcal{M})$. This result confirms the fact that in the FPP case, only one section has to be kept, and in the VPP case not all the sections have to be kept.

\section{SIMULATION RESULTS}

In this section, we compare the performance of different sets of BICM schemes. Experiments are done on the standard 8 bits per pixels (bpp) grayscale $512 \times 512$ Lena picture. The source coder considered is the Jasper JPEG-2000 encoder. The initial picture is encoded at a rate of $0.5 \mathrm{bpp}$. The source is packetized into packets of size $k_{s}=384$ bits.

The RCPC codes taken from [12] have a mother code of rate $1 / 4$ and a polynomial generator $(147,163,135,135)_{8}$ in octal basis. This leads to a set of available channel codes $\mathcal{C}=$ $\{8 / 32,8 / 30,8 / 28, \ldots 8 / 10,8 / 9\}$. The set of the different modulations is $\mathcal{M}=\{4-Q A M, 16-Q A M\}$. For all SNRs and all BICM schemes, the Frame Error Rate (FER) $p_{e}(\mathrm{~cm})$ are computed by Monte-Carlo simulations. The polynomial generator of the 16 bits CRC is $1+X^{2}+X^{15}+X^{16}$. The considered interleaver is random. In the experiments, we consider the following sets: 


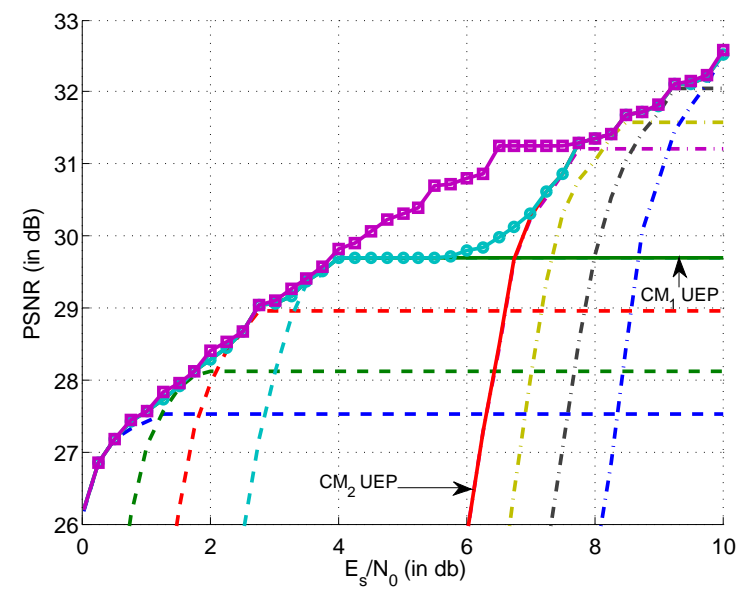

Fig. 3. Comparison of PSNR of all Equal Error Protection (EEP) of set $\mathcal{C M}_{3}$, UEP on $\mathcal{C} \mathcal{M}, \mathcal{C M}_{1}, \mathcal{C M}_{2}$ and $\mathcal{C M}_{3}$. Dash curves represent the different EEP of the $\mathcal{C} \mathcal{M}_{1}$ set. Dash and dots curves represent the different EEP of the $\mathcal{C} \mathcal{M}_{2}$ set. Solid curves represent rate-optimal UEP over the sets $\mathcal{C M}_{1}$ and $\mathcal{C M}_{2}$. Round mark curve represents UEP over the set $\mathcal{C M}_{3}$ and square mark curve represents UEP over the set $\mathcal{C} \mathcal{M}$.

$$
\begin{aligned}
& \text { 1. } \mathcal{C} \mathcal{M}=\mathcal{C} \times \mathcal{M}, \\
& \text { 2. } \mathcal{C M}_{1}=\{(8 / 32,4-Q A M),(8 / 26,4-Q A M), \\
& (8 / 22,4-Q A M),(8 / 18,4-Q A M)\}, \\
& \text { 3. } \mathcal{C} \mathcal{M}_{2}=\{(8 / 24,16-Q A M),(8 / 22,16-Q A M), \\
& \quad(8 / 20,16-Q A M),(8 / 18,16-Q A M)\}, \\
& \text { 4. } \mathcal{C M}_{3}=C M_{1} \cup C M_{2} .
\end{aligned}
$$

The maximum channel rate is 0.125 symbols per pixel (spp), which, for a square picture of size $N$, gives a symbol budget $B_{T}=0.125 N^{2}$ channel symbols. In Fig. 3 we observe that, for low SNR, $4-Q A M$ policies are better while $16-Q A M$ are better for high SNR. From UEP over the sets $\mathcal{C M}_{1}$ and $\mathcal{C M}_{2}$ we observe that the algorithm always chooses to follow the optimal curve and that between those curves, the algorithm uses the diversity of $\mathcal{C M}_{1}$ and $\mathcal{C M}_{2}$ to improve the performance. This phenomenon is similar to an intra-modulation UEP. It is also observed that the PSNR-curve obtained with the set $\mathcal{C M}_{3}$ is at least as good as the maximum of the PSNR-curve for $\mathcal{C M}_{1}$ and $\mathcal{C M}_{2}$ (as proposed by [10]). This phenomenon is similar to an inter-modulation UEP. To give a better observation of inter-modulation UEP, we have reported the proportion of $16-Q A M$ modulation use for the different policies obtained at the different SNR. Notice also that increasing the set $\mathcal{C M}$ yields less mixed policies as it can be interpreted from Fig. 4.

\section{CONCLUSION}

In this work, we have presented a new trellis representation which can be easily derived in many contexts such as VPP contexts or FPP contexts and extended to BICM, parallel channels or HARQ. We have also presented a new algorithm that uses the trellis structure to find a rate optimal policy. The proposed algorithm is linear in the number of reachable policies and linear in the size of the set $\mathcal{C M}$. The advantage of this structure is its flexibility. It allows to switch easily from a VPP context to an FPP context.

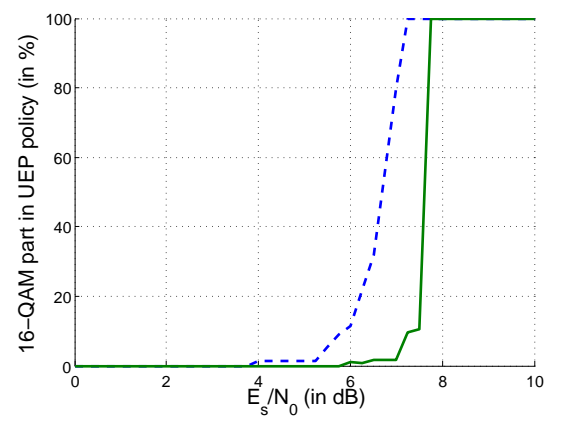

Fig. 4. Comparison of the part of $16-Q A M$ BICMs in the rate allocation policies for different SNR for the sets $\mathcal{C M}$ (solid line) and $\mathcal{C M}_{3}$ (dashed line).

\section{REFERENCES}

[1] P. G. Sherwood and K. Zeger, "Progressive image coding for noisy channels," IEEE Signal Proc. Letters, vol. 4, no. 7, pp. 189-191, July 1997.

[2] A. Said and W. A. Pearlman, "A new fast and efficient image codec based on set partitionning in hierarchical trees," IEEE Tr. Circuits Syst. Video Technol., vol. 6, pp. 243-250, June 1996.

[3] D. Taubman and M. Marcellin, JPEG-2000: Image Compression Fundamentals, Standards, and Practice, Norwell and MA: Kluwer, 2001.

[4] V. Chande and N. Favardin, "Progressive transmission of image over memoryless noisy channels," IEEE J. Select. Areas Com., vol. 18, no. 6, pp. 850-860, June 2000.

[5] V. Stanković, R. Hamzaoui, and D. Saupe, "Fast algorithm for rate-based optimal error protection of embedded codes," IEEE Tr. On Com., vol. 51, no. 11, pp. 1788-1795, Nov. 2003.

[6] R. Hamzaoui, V. Stanković, Z. Xiong, "Fast algorithm for distortion-based error protection of embedded image codes," IEEE Tr. On Image Proc., vol. 14, no. 10, pp. 1417-1421, Oct. 2005.

[7] B. A. Banister, B. Belzer, and T. R. Fisher, "Robust image transmission using jpeg2000 and turbo-codes," IEEE Signal Proc. Letters, vol. 9, no. 4, pp. 117-119, Apr. 2002.

[8] A. Norastina, J. Lu, and B. Aazhang, "Source-channel rate allocation for progressive transmission of images," IEEE Tr. on Com., vol. 51, no. 2, pp. 186-196, Feb. 2003.

[9] L. Pu, M. W. Marcellin, I. Djordjevitc, B. Vasic, A. Bilgin, "Joint source-channel rate allocation in parallel channels," IEEE Tr. on Im. Proc., vol. 16, no. 8, pp. 2016-2022, Aug. 2007.

[10] Y. Sun and Z. Xiong, "Progressive image transmission over space-time coded ofdm-based mimo systems with adaptive modulation," IEEE Tr. on Mobile Computing, vol. 5, no. 8, pp. 1016-1028, Aug. 2006.

[11] J. Qiu, A. H. Banihashemi, "Policy allocation for transmission of embedded bit streams over noisy channels with feedback," IEEE Tr. on Com., vol. 57, no. 11, pp. 3177-3182, Nov. 2009.

[12] J. Hagenauer, "Rate-compatible punctured convolutional codes (RCPC Codes) and their applications," IEEE Tr. on Com., vol. 36, no. 7, pp. 389-400, Apr. 1988. 